\title{
Traces, bâtons à fouir et dessins sur le sable
}

Chez les Aborigènes du Désert occidental australien

Traces, digging sticks and sand drawings among Aboriginal people of the Australian Western Desert

\section{Sylvie Poirier}

\section{Q OpenEdition Journals}

Édition électronique

URL : https://journals.openedition.org/tc/7252

DOI : $10.4000 /$ tc. 7252

ISSN : 1952-420X

\section{Éditeur}

Éditions de l'EHESS

\section{Édition imprimée}

Date de publication : 15 décembre 2013

Pagination : 144-165

ISBN : 978-2-7351-1654-6

ISSN : 0248-6016

\section{Référence électronique}

Sylvie Poirier, «Traces, bâtons à fouir et dessins sur le sable», Techniques \& Culture [En ligne], 61 | 2013, mis en ligne le 15 décembre 2016, consulté le 29 septembre 2022. URL : http:// journals.openedition.org/tc/7252 ; DOI : https://doi.org/10.4000/tc.7252 


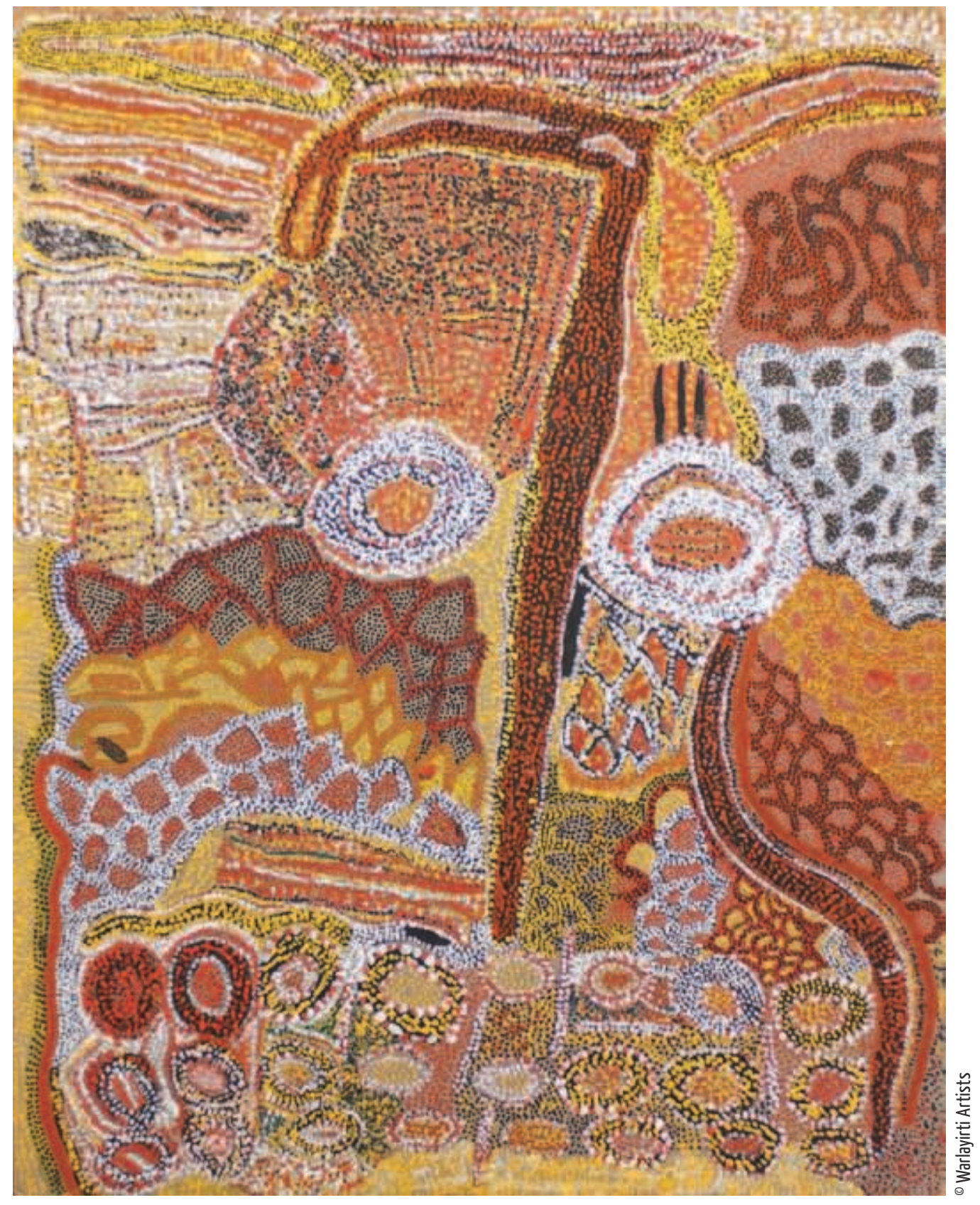


Sylvie Poirier

Université Laval

Sylvie.Poirier@ant.ulaval.ca
Vivre le sable! Corps, matière et sociétés - 2

Techniques \& Culture 61, 2013/2 : 144-165

\section{TRACES, BÂTONS À FOUIR ET DESSINS SUR LE SABLE}

\section{Chez les Aborigènes du Désert occidental australien}

La communauté aborigène de Balgo (ou Wirrimanu) est située aux confins nord du désert de Gibson en Australie occidentale ${ }^{1}$. Le désert de Gibson forme, avec les Grand et Petit déserts de sable et le Grand désert de Victoria, un ensemble culturel, géographique et écologique dénommé le Désert occidental. La communauté de Balgo compte aujourd'hui une population fluctuante d'environ cinq cents personnes. La plupart des familles aborigènes qui y vivent sont originaires des régions désertiques situées plus au sud, un « pays » de sable et de spinifex. Parmi les groupes représentés, les Kukatja et les Walmatjari sont en majorité ; y vivent aussi des Wangkatjunga, des Mandiltjarra, des Tjaru, des Ngarti et des Pintupi ${ }^{2}$.

Traditionnellement chasseurs-cueilleurs nomades au sein d'un environnement dominé par les plaines et les dunes de sable, et sédentarisés à partir des années 1950, les Kukatja et les groupes voisins maintiennent avec leurs territoires ancestraux une relation intime, dont ils sont, suivant la Loi ancestrale (Tjukurrpa), les gardiens dépositaires. Cette relation s'exprime aussi par le biais des activités de chasse et de cueillette qu'ils pratiquent encore aujourd'hui, quoique de manière irrégulière. Dans ce texte, nous soulignerons à la fois la diversité et la rareté des ressources animales et végétales de cet environnement où domine le sable et présenterons l'étendue du savoir aborigène, notamment en ce qui a trait à la chasse au petit gibier et à la cueillette, à l'art de pister sur le sol sablonneux mais aussi de creuser, aux techniques associées à ces pratiques et à l'outil féminin par excellence, le bâton à fouir (wana) - autrefois de bois, aujourd'hui de métal. Autant de pratiques auxquelles nous ont introduite les Aborigènes de Balgo lors de très nombreuses sorties 
en brousse. Enfin, nous évoquerons l'art de raconter sur le sable et présenterons brièvement des expressions artistiques contemporaines des Aborigènes, inspirées de leur relation avec le sable et l'environnement désertique.

\section{« Pays » de sable et de spinifex}

Quoique désertique, le territoire des Kukatja et des groupes voisins n'en présente pas moins une certaine diversité. La région est dominée à $75 \%$ par les plaines sablonneuses et les dunes de sable (tjilytji ou tali), ces dernières d'une hauteur moyenne de vingt mètres et orientées suivant les vents dominants de l'est. Plaines et dunes sont recouvertes de spinifex, une graminée endémique des régions désertiques d'Australie qui forme des touffes d'herbe épineuse. Autrefois, le spinifex était utilisé à plusieurs fins par les Aborigènes : la graine était récoltée, moulue et cuite en pain dans les cendres; durant la saison chaude, ils en extrayaient la résine, utilisée une fois refroidie pour la fabrication et la réparation de leurs outils de bois. Les plaines sablonneuses sont aussi parsemées de termitières, d'une hauteur moyenne d'un mètre. Le sable durci des termitières servait autrefois à divers usages thérapeutiques (Peile 1997).

On trouve aussi des plaines au sol plus argileux où poussent, entre autres, plusieurs espèces d'acacia. Les acacias, dont le « mulga » (Acacia aneura, son bois est prisé pour sa dureté), fournissaient, notamment, les bois préférés pour la fabrication des différents outils et armes (boomerangs, boucliers, lance-javelots, gourdins, et les récipients utilisés par les femmes ${ }^{3}$ ). De rares formations rocheuses et quelques chaînes montagneuses

Formation rocheuse au sud de Balgo identifiée à Lurnn (Homme Martin-Pêcheur)

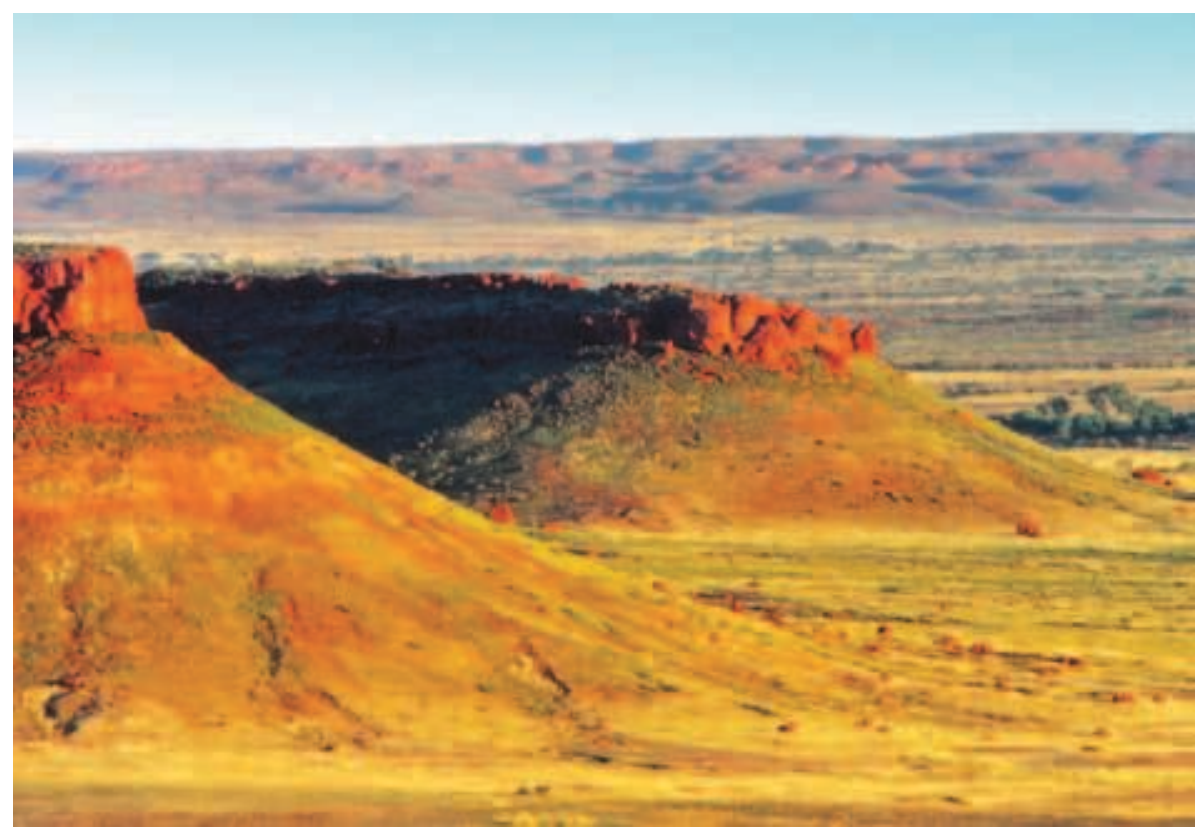




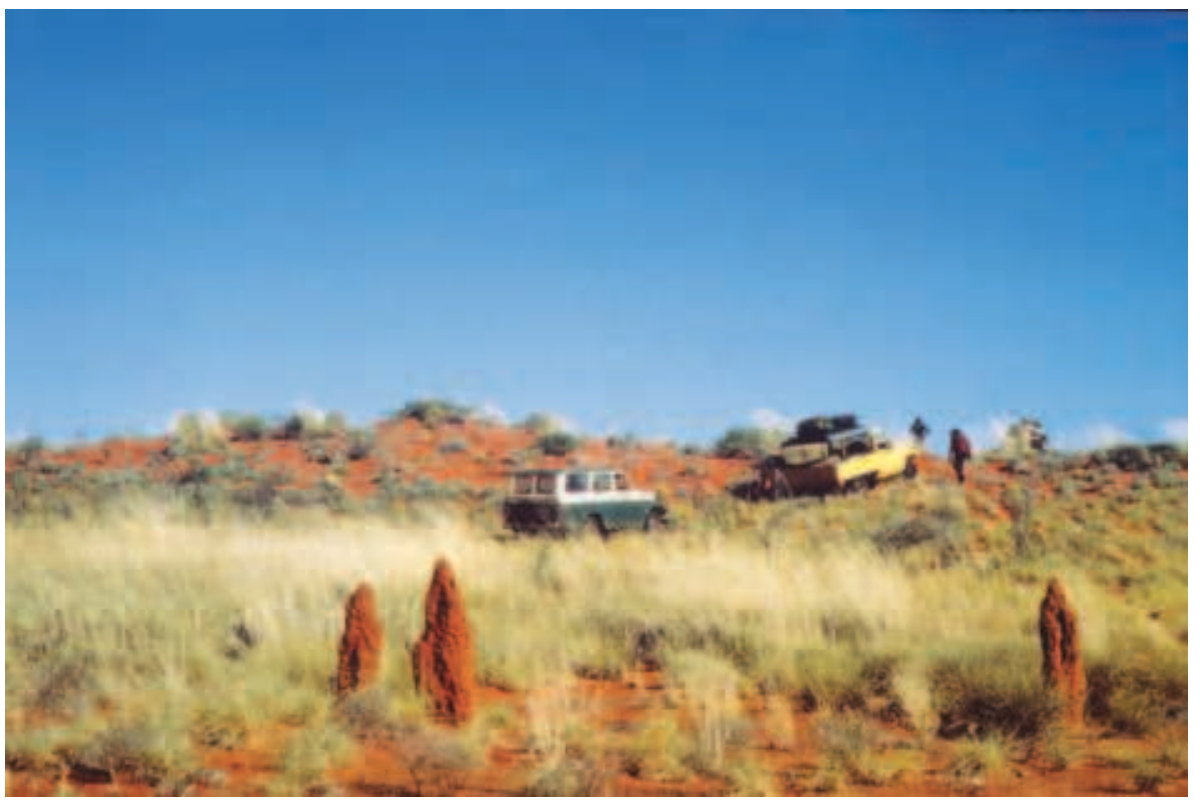

ponctuent le paysage ici et là. Sur l'étendue du Désert occidental, on rencontre des lacs de sel de superficies variées. On trouve aussi des cuvettes de sol argileux (« claypan » en anglais australien), des criques plus ou moins vastes, asséchées la majeure partie de l'année, et le long desquelles poussent de grands eucalyptus.

Sur un cycle annuel, les Aborigènes du Désert occidental distinguent trois saisons (voir aussi Walsh 1990). De mars à août c'est la saison froide (yaltapuru), avec des températures moyennes de $25^{\circ} \mathrm{C}$ le jour, pouvant descendre à $5^{\circ} \mathrm{C}$ la nuit. Cette période fait suite aux pluies bienfaitrices : les graines et les larves sont abondantes alors que les reptiles hibernent en s'enfouissant dans le sable. La période d'août à octobre, analogue au printemps (tuulkarra), est la plus sèche, des nourritures végétales sont encore disponibles et les reptiles refont surface. D'octobre à mars, c'est la saison chaude (yarlipuru), avec des températures pouvant atteindre $45^{\circ} \mathrm{C}$ à l'ombre ; les pluies sont plus abondantes à partir de janvier, les fèves d'acacia sont matures et c'est une période propice pour la chasse aux gros et petits gibiers.

D’une année à l'autre, les pluies sont très irrégulières, voire incertaines. On parle d'une moyenne annuelle de précipitations de $200 \mathrm{~mm}$. Durant la saison des pluies, la plaine désertique peut rapidement se transformer en une mer intérieure le temps de quelques heures ; l'eau étant rapidement absorbée par le sol sablonneux et évaporée par un soleil cuisant. Au temps du nomadisme, durant la saison chaude, il était fréquent que les Aborigènes creusent un trou dans les dunes de sable afin de s'y enfouir et de se protéger ainsi de la chaleur intense. Outre le sable, d'autres éléments naturels doivent être mentionnés, en ce qu'ils participent de la relation aborigène à cet univers désertique.

Dans ces vastes plaines sablonneuses, le vent (warlpa) est une présence quasi-permanente. Durant la journée, il peut souffler de manière régulière et continue, ou alors prendre la forme de mini-tornades. Dans les deux cas, quelle que soit leur intensité, les vents soulèvent et transportent le sable, si bien que le corps humain est constamment en contact avec lui, qui recouvre la peau et s'immisce dans les yeux, les narines et les oreilles. 


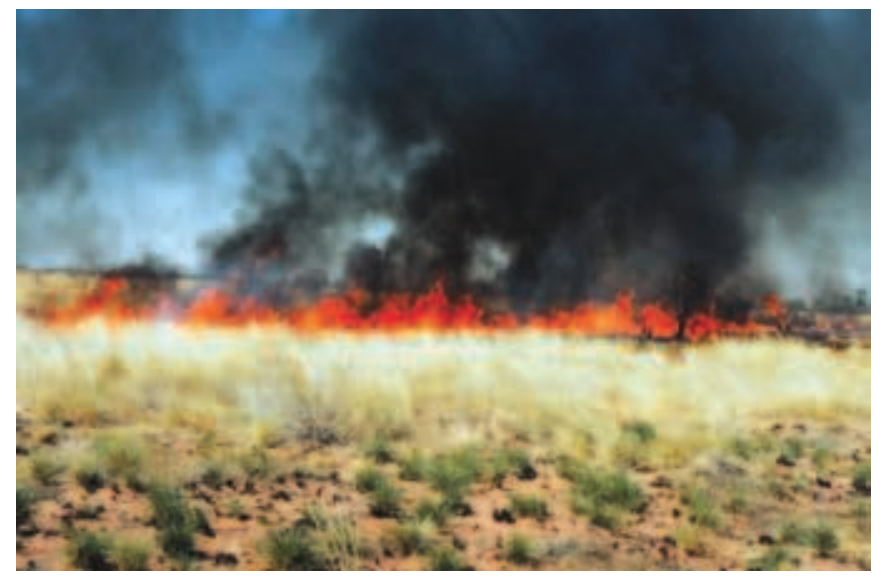

Ci-dessus et dessous. Technique de brûlis en mosaïque

Afin de promouvoir la régénération de la végétation et de faciliter la chasse au petit gibier,

les Aborigènes pratiquent la technique ancestrale de brûlis en mosaïque.

2006

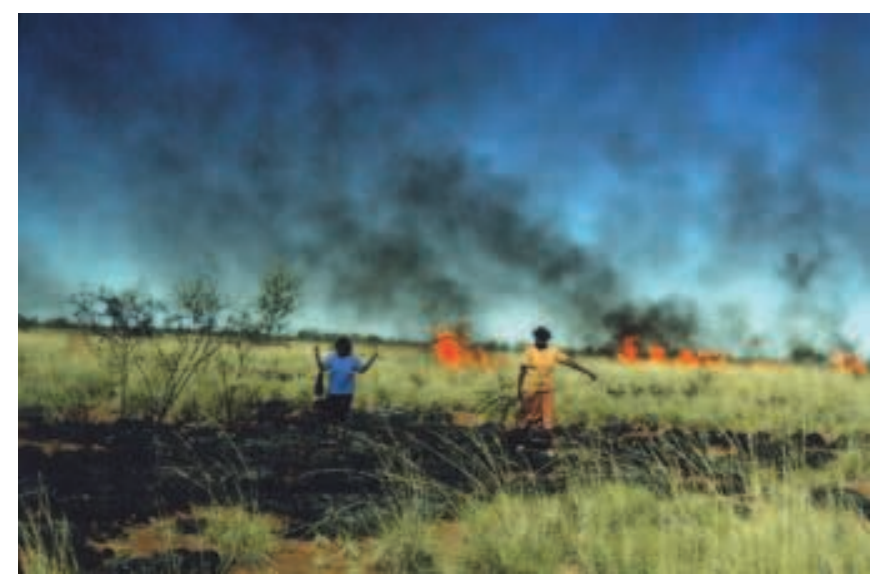
ne recouvre la nourriture.
Les Aborigènes ne semblent aucunement incommodés par ces présences, et laissent le vent et le sable pénétrer par tous leurs pores. En revanche lorsque le vent et le sable se mêlent en mini-tornades qui se dirigent vers les campements, ils tentent de faire dévier leur trajectoire par des chants et des gestes rituels.

Au temps du nomadisme, le corps nu des Aborigènes était ainsi constamment recouvert d'une mince couche de sable laquelle les protégeait du soleil. Durant la saison chaude ou après un effort physique, il leur arrive encore de se frotter avec du sable afin d'atténuer la sueur et la chaleur du corps. Afin de se protéger du vent et du sable, les camps temporaires d'autrefois comportaient nécessairement un coupe-vent (kapati) fabriqué avec des branchages et du spinifex. Encore aujourd'hui, lors des sorties en brousse, ils construisent de tels coupe-vents afin de se protéger et d'éviter qu'une couche de sable

Le feu (waru, un terme générique) est aussi très présent. Les Aborigènes pratiquent depuis toujours une technique de brûlis en mosaique qui vise notamment le contrôle et la régénérescence de la végétation et permet d'attirer le gibier ${ }^{4}$. Cette technique permet de dégager une étendue de spinifex, facilitant ainsi la traque et la chasse au petit gibier (Cane, Stanley 1985 ; Vaarzon-Morel, Gabrys 2009 ; Bird et al., 2005).

L'eau (kalyu, ngapa) est bien entendu une ressource rare. On trouve des points d'eau extérieurs, encastrés dans les rochers et de volumes divers. L'eau y est plus ou moins permanente, selon leur profondeur et les précipitations au fil des ans. Mais dans la majorité des cas, les points d'eau, sources et autres, sont souterrains et présentent un caractère plus permanent. Les « soakage », en anglais australien, sont connus des seuls Aborigènes; un œil inexpérimenté ne serait pas en mesure de les détecter. Pour accéder à ces ressources, les Aborigènes creusent le sol sablonneux parfois jusqu'à plusieurs mètres de profondeur.

Les sorties en brousse et les activités de chasse et de cueillette nécessitent maintenant des véhicules tout-terrain. En effet, les Aborigènes doivent s'éloigner de la communauté de quelques dizaines de kilomètres afin de trouver des régions propices à la chasse et à la cueillette. Depuis les années 1980, au cours de mes séjours dans la région, j’ai ainsi régulièrement accompagné mes amis aborigènes lors de ces sorties et ai apprécié l'étendue de leurs connaissances.

Dans le Désert occidental, les ressources et nourritures animales ( $k u k a$ ) et végétales (mangarri) sont rares et très certainement éparses, mais elles n'en sont pas moins diversifiées ${ }^{5}$. À l'instar du gros gibier - kangourou, émeu, outarde australienne, bandicoot et wallaby -, le petit gibier est encore régulièrement chassé ; il est aussi plus abondant que le premier. Le petit gibier comprend plusieurs espèces de lézards et de serpents et de petits marsupiaux tels les opossums, les 
porcs épics (échidnés) et les quolls (dasyure ou chat marsupial). Durant la saison froide, les reptiles s'enfouissent dans le sable pour hiberner. Les différentes sortes de grenouilles, généralement enfouies sous le sable, sont aussi un mets fort apprécié. Certaines espèces sont récoltées en creusant le long des parois sablonneuses des criques asséchées. Une espèce de grenouilles des sables (purrpurrpa) est récoltée quant à elle sur le sommet des dunes durant la saison sèche. Il est alors tout à fait impressionnant de voir les Aborigènes creuser dans le sable des trous de plusieurs mètres de largeur et de profondeur afin de récolter des quantités étonnantes de purrpurrpa ; elles seront ensuite grillées sur le feu allumé à proximité.

Au fil des saisons et considérant les conditions désertiques, la diversité des ressources végétales comestibles est étonnante (Walsh 1990). Parmi celles-ci, les graines de différentes herbes et les fèves d'acacia, lesquelles demandaient un temps de cueillette et de préparation appréciable, ne sont plus collectées ${ }^{6}$. Au temps du nomadisme, lors d'une saison particulièrement abondante, graines et fèves étaient stockées, soit dans des trous creusés dans le sol sablonneux et que l'on tapissait de spinifex, soit dans des cavités rocheuses, en prévision de périodes de disette (Cane 1984).

Plusieurs ressources végétales sont encore collectées et consommées. De mars à octobre, c'est le temps des tubercules dont il existe plusieurs variétés. La plus répandue et recherchée est l'igname du désert, le karnti, dont nous parlerons plus loin. C'est aussi le temps des tjunta (les « bush-onions »). Très petits, ils sont récoltés en grande quantité en dégageant du revers de la main le sol sablonneux. On les trouve surtout à proximité des zones argileuses. Les tjunta sont ensuite légèrement rôtis en étant déposés sur une couche de sable chaud dégagé à proximité du feu. D'août à décembre, c'est la saison des fruits sauvages. Parmi ceux-ci, les kumpupatja (« bush-tomato », de la famille des solanacées) sont particulièrement populaires

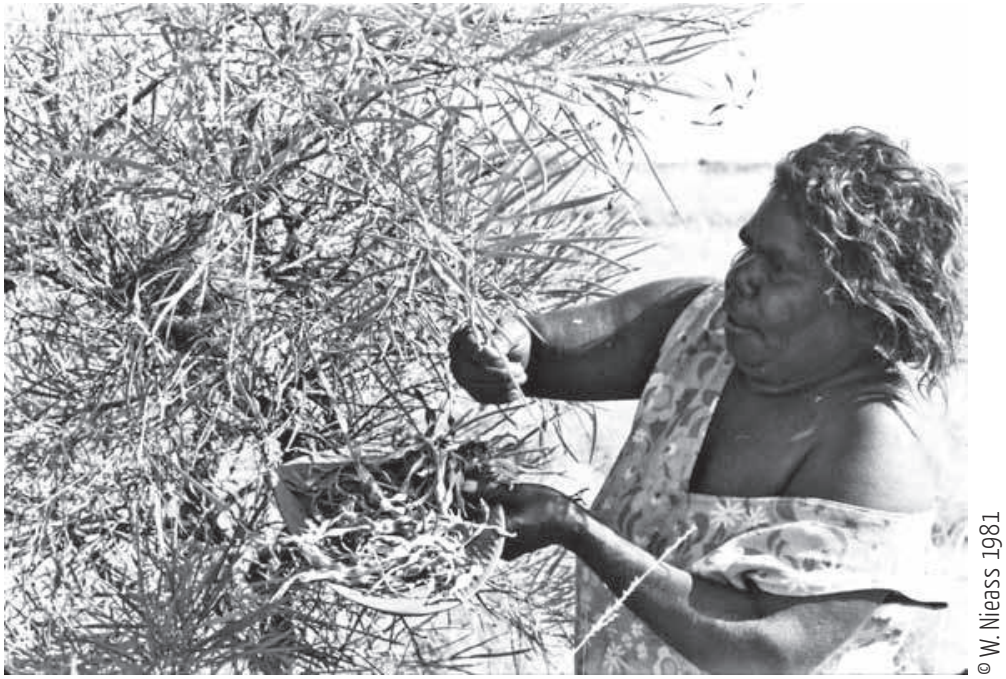

Cueillette de fèves d’acacia ainsi que les kantjilyi (« bush-raisin », de la même famille).

Lors des sorties en brousse, le gros et le petit gibiers ainsi que les nourritures végétales, comme les tubercules par exemple, sont cuits à la manière traditionnelle. On allume d'abord un feu, avec des brindilles et du bois mort ; lorsque le feu s'est consumé, laissant un lit de braises rougeoyantes, un trou est creusé à proximité. Le fond du trou est tapissé de braises chaudes ; on y dépose alors le gibier, préalablement éviscéré, ou le tubercule, que l'on recouvre ensuite d'un mélange de braises et de sable chaud. La grosseur du trou, dans un sol meuble et sablonneux, ainsi que le temps de cuisson varient selon que l'on prévoie de cuire un émeu ou un serpent!

Il s'agit bien entendu d'un rapide survol de l'ensemble des produits chassés, cueillis et consommés dans cette région du Désert occidental. Les Aborigènes du Désert occidental sont ainsi passés maîtres dans l'art de creuser le sol sablonneux à la recherche d'eau, de tubercules, de lézards, de serpents ou de grenouilles. Nous y reviendrons. 


\section{Traces et tracés Tjukurrpa}

Cet environnement aride et sablonneux, aux humeurs incertaines, n'en a pas moins inspiré aux Aborigènes une riche tradition cosmologique, narrative et rituelle. Dans leur perspective, connaître un territoire, l'habiter et le parcourir, c'est aussi en connaître les ressources spirituelles, intimement liées au milieu écologique et aux ressources matérielles. Les anthropologues et autres observateurs des mondes aborigènes ont beaucoup écrit sur ce qu'il est maintenant convenu d'appeler le Dreaming ou le Rêve. J'utiliserai ici le terme en langue vernaculaire des Aborigènes de Balgo, soit Tjukurrpa.

Le Tjukurrpa est un concept plurivoque et pluridimensionnel (Stanner 1979); il désigne simultanément une cosmologie, une ontologie, une cosmogonie et une épistémologie. Le Tjukurrpa, que les Aborigènes traduisent aussi par « Law », est au fondement de la compréhension et de l'expérience aborigènes du monde; en tant qu'essence fondatrice, il est la clé de la reproduction dynamique de tout ce qui est : humain, animal, végétal, minéral, ainsi que les essences ancestrales semées par les êtres Tjukurrpa. Pour les Aborigènes, l'univers est la création initiale des êtres Tjukurrpa. Investis de pouvoirs suprahumains, ces êtres, masculins et féminins,

Piste d'un serpent

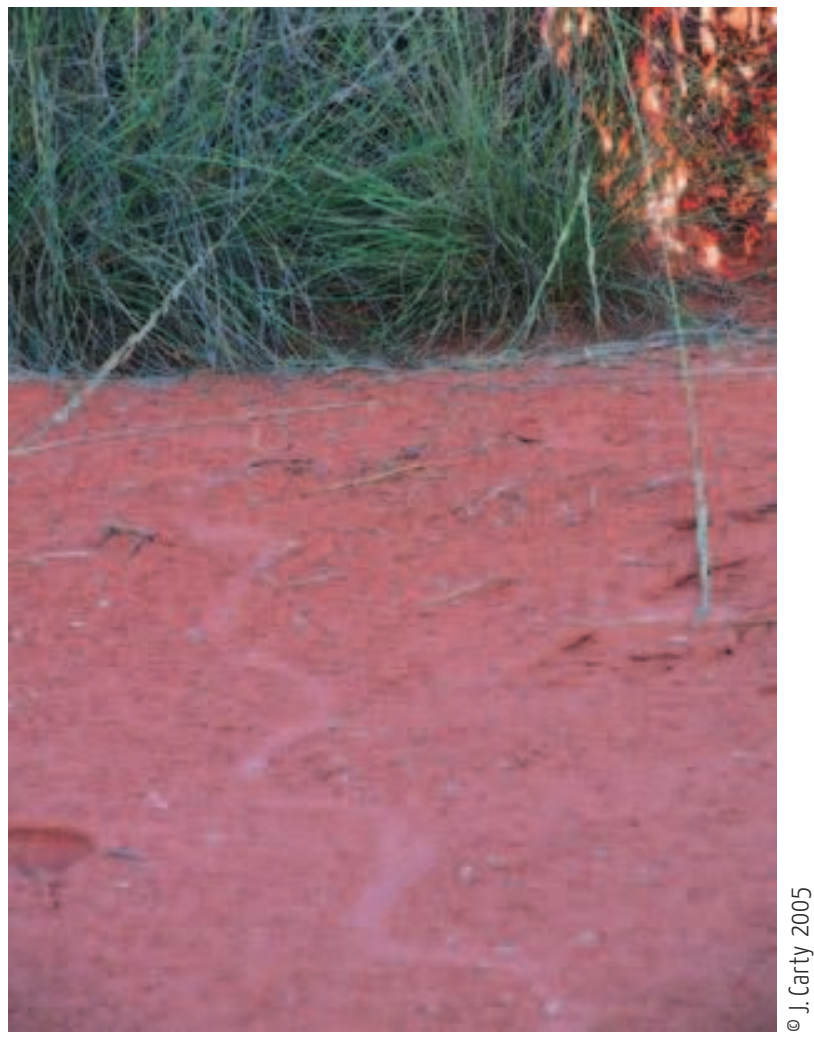

ont voyagé sur la surface de la terre, dans le monde souterrain et dans les cieux. Ils ont tantôt forme humaine, tantôt forme animale ou végétale, ou celle encore des éléments naturels dont ils portent les noms.

Au fil de leurs pérégrinations, les êtres Tjukurrpa ont façonné le paysage tel qu'on le connaît aujourd'hui et nommé l'ensemble des lieux, des espèces animales et végétales, des corps célestes ; ils ont semé sur leur passage les essences reproductrices, les esprits-enfants, dont les humains sont des incarnations. Les traces de leur passage sont à jamais présentes sur le territoire, inscrites à même le paysage. Les êtres Tjukurrpa continuent de s'incarner et de s'actualiser dans le monde actuel, par le biais entre autres des esprits-enfants, ou de se révéler en rêve aux hommes et aux femmes (Dussart 2000, Myers 1986, Poirier 1996).

Chaque lieu et configuration du paysage sont ainsi identifiés à un ou des êtres Tjukurrpa, à leurs actes ou à leurs corps métamorphosés (mais aussi à leurs substances corporelles, comme le sang, l'urine, le gras ou le sperme, ou à leurs objets, tels les boomerangs, javelots, boucliers ou bâtons à fouir). Encore aujourd'hui, lorsqu'ils se promènent au sein des plaines désertiques, à pieds ou en véhicule motorisé, et afin d'expliquer 
la configuration physique des lieux, les Aborigènes ne sont jamais à court de ces récits merveilleux et dramatiques mettant en scène les êtres Tjukurrpa. Telle marque sur le sable durci est l'empreinte laissée pas Lurrn, l'Homme-Martin-Pêcheur, alors qu'il se dirigeait vers le sud ; tel point d'eau est l'endroit où les Kanaputa, les Femmes-Bâtons-à-fouir, ont pénétré le sol, après avoir dérobé les objets-sacrés et ainsi échappé à leurs poursuivants (Poirier 1996: 52). Les récits qui relatent de la sorte les itinéraires des êtres Tjukurrpa comportent des toponymes, des informations sur les ressources animales et végétales du territoire parcouru ou encore sur les points d'eau. Ils tiennent le rôle en quelque sorte de «cartes cognitives».

Une série de lieux identifiés à un être Tjukurrpa délimite à son tour des aires d'affiliation et de responsabilité rituelle; les Aborigènes parlent alors de leur «pays» (ngurra). À chaque «pays » correspondent des récits ainsi que des savoirs mythiques et rituels (chants, danses, dessins) légués par les êtres Tjukurrpa, et dont sont dépositaires et gardiens les hommes et les femmes affiliés au lieu en question ${ }^{7}$. Les séquences et danses rituelles recréent ainsi les actions et les voyages des êtres du Rêve. Sur les scènes rituelles, de jour ou de nuit, les pieds nus des groupes de danseurs, hommes ou femmes, glissent en pas saccadés sur le sol sableux, soulevant un nuage pulvérulent qui reste en suspension (dans les airs), intensifié tantôt par les rayons du soleil, tantôt par la lueur des flammes.

Du point de vue des Aborigènes, l'ensemble du Désert occidental se déploie donc telle une gigantesque fresque narrative où se jouent des drames immémoriaux. Les humains sont partie prenante de ceux-ci. Non seulement ils les actualisent dans les mises en scène rituelles, mais ils y réfèrent dans leur vie quotidienne (Poirier 2004). Parcourir les plaines sablonneuses en compagnie des Aborigènes ne peut jamais être banal ou monotone. Une mini-tornade peut être interprétée comme la présence d'un être Tjukurrpa ; un iguane tué sera associé à l'enfant à naître d'une des femmes du groupe (on dira que l'esprit-enfant avait momentanément pris la forme de l'iguane) ; en passant près d'une formation rocheuse, un adulte fera remarquer que nous passons à proximité de la demeure de tel parent défunt.

\section{L'art de pister et de creuser}

On comprend mieux maintenant comment pour les Kukatja et les groupes voisins les savoirs écologiques, géographiques, hydrologiques, mythiques et rituels sont étroitement interconnectés et à plusieurs égards s'informent mutuellement. Connaître un territoire, c'est aussi savoir reconnaître toutes les traces qui y sont inscrites, de manière permanente ou évanescente. Hormis les traces permanentes laissées par les êtres Tjukurrpa, il faut également être en mesure d'identifier les traces et les empreintes des humains, des animaux et des végétaux. Le sol sablonneux se révèle, au regard aguerri des Aborigènes, un véritable livre à ciel ouvert où se côtoient, se croisent et s'entrecroisent les pistes des uns et des autres, que seul un œil attentif et expérimenté est en mesure de décoder. La notoriété des Aborigènes, pisteurs émérites, n'est plus à faire. Ils possèdent en effet l'art de lire et d'interpréter les traces et 
empreintes diverses, celles laissées par les humains et les véhicules motorisés, mais aussi les traces spécifiques de chaque espèce animale, des plus grands gibiers aux plus petits. Mieux encore, ils peuvent souvent reconnaître l'empreinte spécifique de chaque animal, individu ou véhicule appartenant à la communauté. Le terme tjina désigne à la fois les pieds, mais aussi toutes les formes d'empreintes, soientelles animales, humaines, mécaniques, ou ancestrales. Dans la langue kukatja, il existe une multitude d'expressions dérivées du terme tjina afin de qualifier et de désigner soit la forme du pied, une démarche particulière ou bien encore la vélocité (Valiquette 1993).

De nos jours, cette capacité de lire, traduire et interpréter les traces et les empreintes sur le sol sablonneux, et notamment les pistes animales, est un savoirfaire acquis très jeune, qui perdure. Une fois repérée, la piste est décodée : l'espèce animale, la grosseur et le sexe de l'individu, la direction empruntée, le moment de son passage. Même dans un véhicule qui roule en brousse à $40 \mathrm{~km} / \mathrm{h}$ ou davantage, les occupants sont toujours aux aguets, scrutant le sol qui défile, à la recherche d'une piste; il n'est pas rare que les enfants soient les premiers à les déceler. Dès qu'une piste animale est repérée, les Aborigènes en évaluent d'abord la fraîcheur. Dans tous les cas, la nature plus ou moins récente de la trace, commande la pertinence ou non de se tenir à l'affût. La piste fraîche d'un varan, par exemple, est reconnaissable à la manière caractéristique qu'ont les grains de sable soulevés par l'animal de retomber encore dans sa trace. Or, les pistes laissées par le petit gibier comme les lézards ou les serpents ne sont en fait que de fines lignes sur le sable. Elles en disent pourtant long au chasseur expérimenté.

Si la piste est fraîche, le chasseur, homme ou femme, se met instantanément en mouvement. Sachant l'animal à proximité, le pisteur se met à sa poursuite ; celle-ci pourra durer de quelques minutes à plusieurs heures. La personne qui repère la piste, avertit les autres membres du groupe, souvent dispersés mais à portée de vue et de voix, par un cri, un signe ou encore en mimant l'animal par un mouvement du corps significatif. Le terrain plat et dégagé offre la possibilité de courir, en zigzaguant parmi les spinifex, souvent pieds nus, tout en suivant les pistes fraîches du varan. Parfois la piste disparaît, là où le sol est plus dur ; le groupe se disperse alors

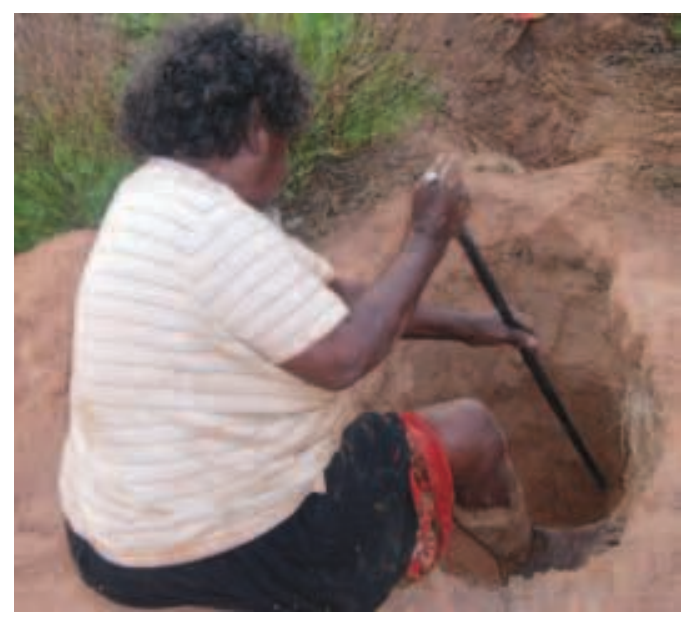

Creuser à la recherche d'un varan 2006

Page ci-contre, aux termes d'une chasse au varan 2006 


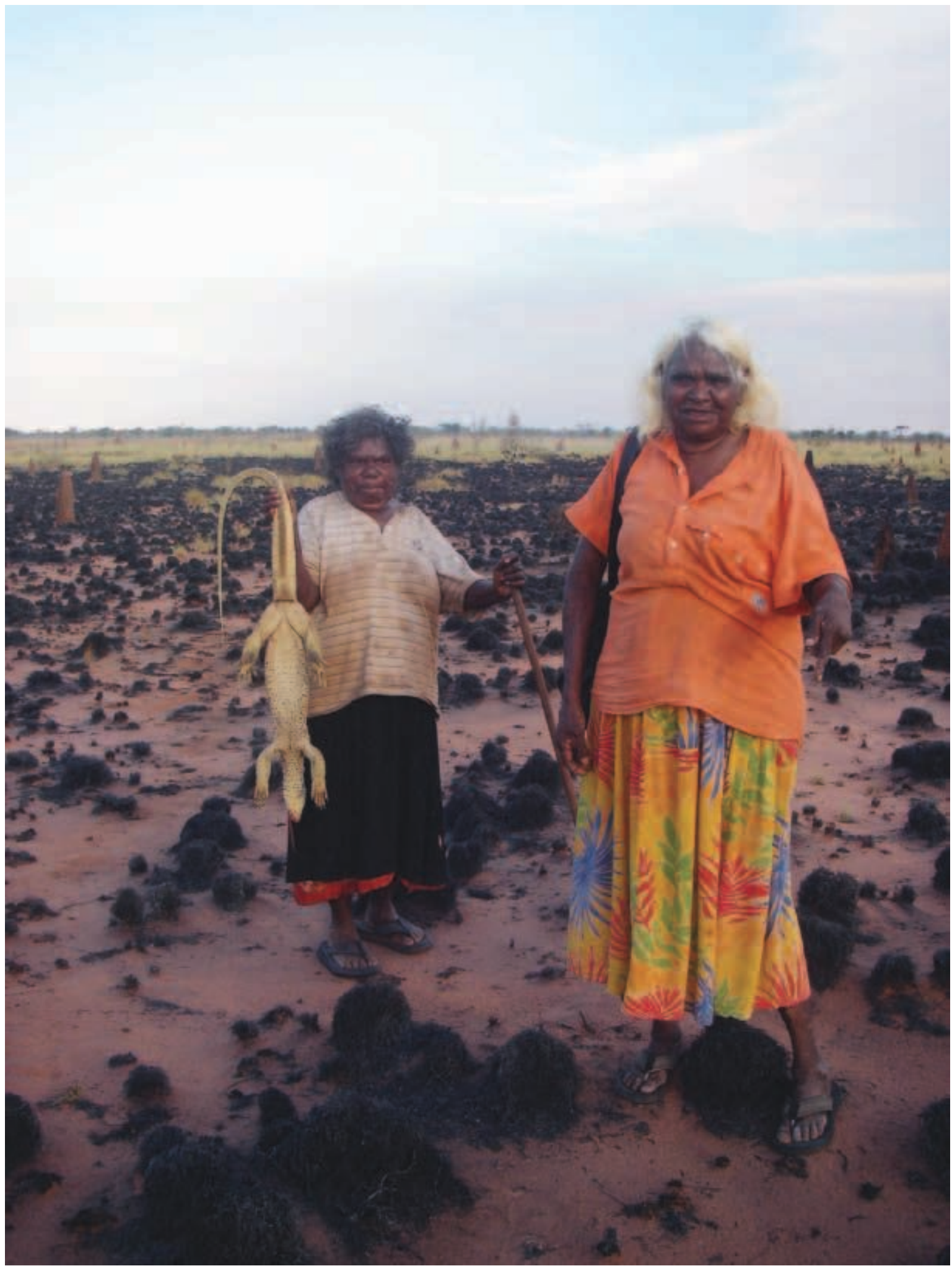



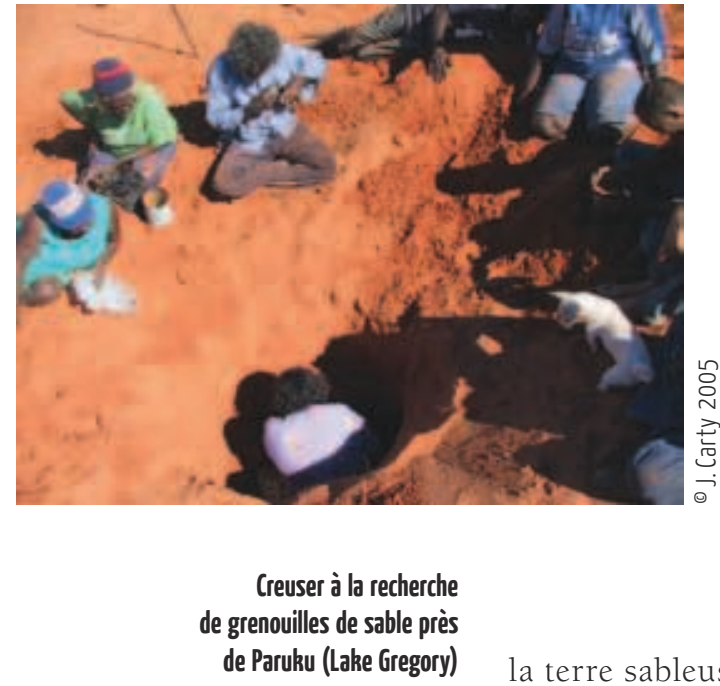

Grenouilles de sable

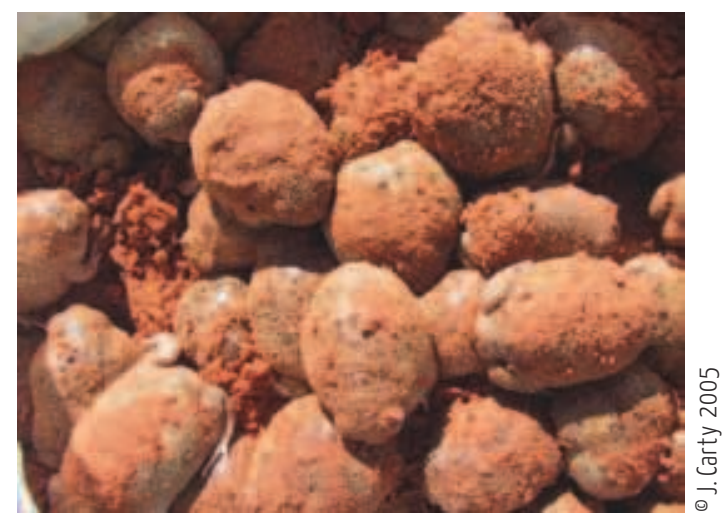

tentant de repérer la piste à nouveau et la direction empruntée par le gibier. Se sentant poursuivi, le varan accélère sa course jusqu'au moment où il choisit de se réfugier dans un trou. Ces trous sont nombreux et donnent accès à des galeries souterraines où vivent les lézards durant les mois froids.

Le terme de la piste révélant l'entrée du trou, la chasse prend une autre forme. Une des femmes s'assoit à l'entrée et commence à creuser le sol sablonneux avec son bâton à fouir (wana), pendant que les autres tâtent, également à l'aide d'un bâton, le sol alentour pour repérer l'emplacement des galeries. Débute un véritable jeu de cache-cache et d'efforts remarquables; plusieurs trous seront parfois creusés avant de parvenir à trouver l'endroit où s'est réfugié le varan. Afin d'éviter les morsures, il faut toujours le prendre par la queue pour le tirer hors du trou.

En plus des pistes animales, d'autres traces s'inscrivent sur la terre sableuse selon le degré de dureté de celle-ci. C'est le cas notamment des craquelures formées à la surface du sol par les tiges souterraines des tubercules. Dans les régions désertiques, l'igname sauvage (karnti) est encore très appréciée et cueillie, approximativement, de mars à octobre. Une fois l'arbuste repéré, les femmes se déplacent autour et dans différentes directions afin de distinguer les fines lignes et les gerçures dessinées par les rhizomes souterrains sur la partie affleurante et durcie du sable. Mais il ne suffit pas de découvrir les traces laissées par la plante, il faut encore évaluer la profondeur et la grosseur du tubercule., Les femmes sondent les fissures, à l'aide de leur wana : la sonorité produite par le contact de l'outil avec le sol leur permet d'évaluer la grosseur de l'igname et sa profondeur. L'igname à présent localisée, un dur labeur commence pour la déterrer, à l'aide du wana, du luantja (récipient aux bords recourbés, en bois ou en métal, utilisé par les femmes) ou de tout autre récipient ; les trous ainsi creusés peuvent atteindre plusieurs mètres de diamètre et de profondeur.

À l'art de pister succède celui de creuser. Assis à même le sol, les jambes croisées devant ou repliées sous lui, le pisteur ramollit le sol sablonneux avec son bâton à fouir, dégage le sable à l'aide de ses mains ou d'un récipient, frappe le sol à nouveau, dégage le sable, et ainsi de suite, alors que son buste se penche toujours un peu plus profondément dans le trou. Les gestes et les mouvements sont souples, vigoureux et répétitifs. La personne garde le silence, tout entière absorbée par cette action qui prend par moments l'allure d'une chorégraphie. Parfois, un autre individu prendra la relève. Il arrive que tout son corps disparaisse dans le trou ainsi creusé à même le sable. Le travail est éreintant, pourtant ces femmes et ces hommes peuvent passer des heures à creuser le sol sablonneux à la recherche de tubercules ou de reptiles (lézards, serpents ou grenouilles), souvent sous des chaleurs écrasantes. En règle générale, ce sont les hommes qui sont responsables de creuser aux points d'eau souterrains.

Cette large présence des trous, qu'ils soient creusés par les humains ou les animaux, celle aussi des galeries et des points d'eau souterrains sont des réalités incontournables sur toute 


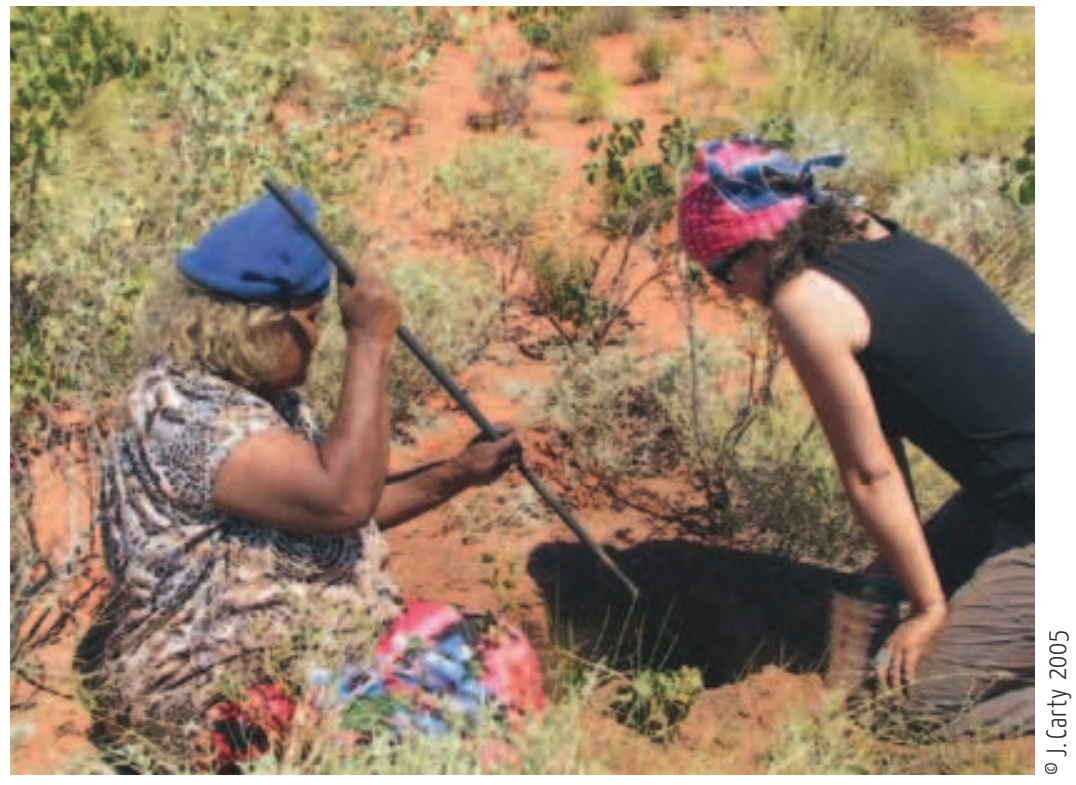

Creuser à la recherche de tubercules

l'étendue du Désert occidental. Les récits Tjukurrpa y font d'ailleurs souvent référence, plus précisément en ces lieux, durant leurs pérégrinations, où les êtres ancestraux ont pénétré dans le sol et voyagé sous terre, avant de resurgir plus loin. Ces segments mythiques sont des références explicites à la vie et à l'activité souterraines.

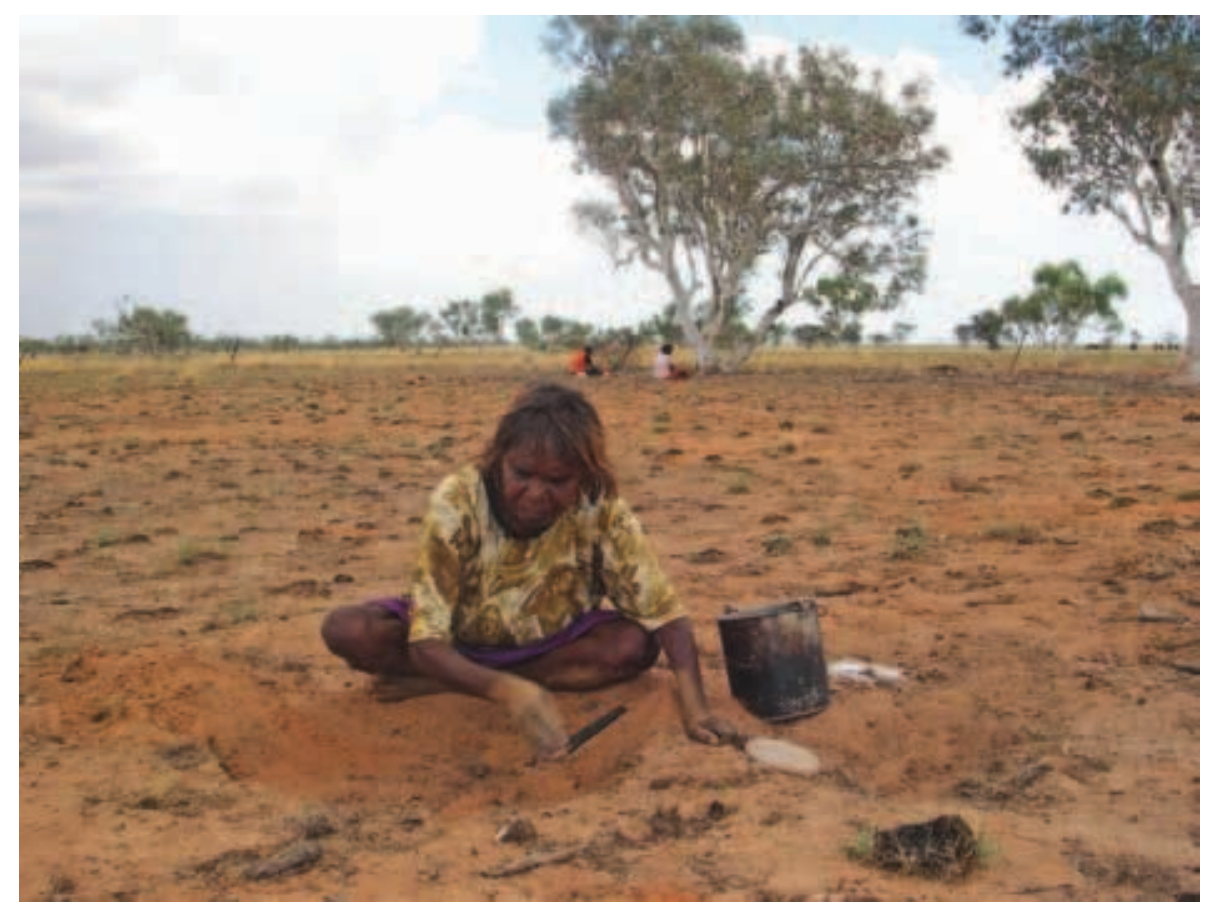

Cueillette des tjunda

(«bush onions») 


\section{L'art de raconter sur le sable}

Dans les traditions orales, comme celle des Kukatja, tout est prétexte à raconter et à être partagé sous forme de récits performatifs. Chaque événement, inusité ou quotidien, dramatique ou comique, est susceptible d'être transformé en récit et prend la forme dès lors d'un événement narratif (Bauman 1986). Raconter est une pratique et un art hautement valorisés et joue un rôle essentiel dans le processus de socialisation des enfants ainsi que dans la transmission des savoirs (rituels, mythiques, écologiques, familiaux ou historiques). J'ai mentionné déjà la richesse et la multiplicité des récits Tjukurrpa et des épisodes narrant les voyages des protagonistes Tjukurrpa, comme une façon de raconter les lieux et le paysage. Or, une des composantes essentielles à toute performance narrative, et non seulement aux récits Tjukurrpa, est son expression graphique.

Les dessins sacrés issus du Tjukurrpa, les kuruwarri, ne sont pas que de simples repré-

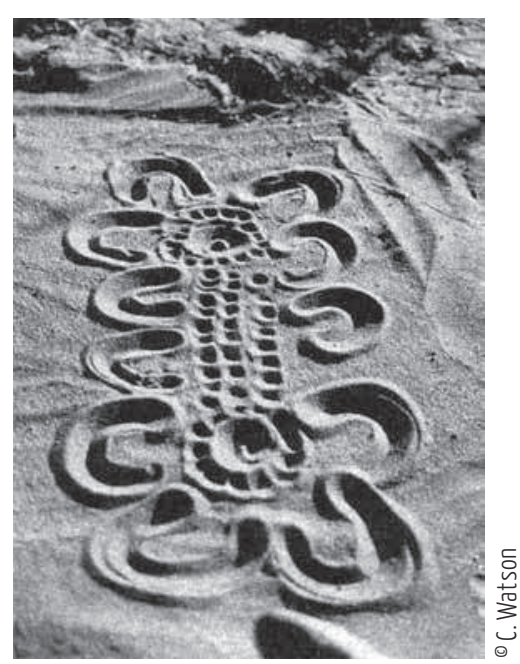
sentations, ils sont une manifestation de la présence et du pouvoir des êtres Tjukurrpa, et rappellent l'interconnexion entre le Tjukurrpa, les humains et les lieux du territoire. Lors des performances rituelles, ces dessins sont appliqués sur les corps et les objets cultuels. Traditionnellement, lors de certains rituels, les Aborigènes effectuaient aussi des peintures sur le sol, sur un espace de sable durci (« ground painting »). Les matériaux utilisés étaient les mêmes que pour les peintures corporelles, soit des pierres d'ocre (aux couleurs variant du rouge au jaune $)^{8}$, du kaolin et du charbon de bois, broyés et mélangés à du gras animal (ou de l'huile de cuisson pour les peintures corporelles) ainsi que du duvet sauvage (blanc ou enduit d'ocre rouge). La peinture sur le sol devenait la scène de la danse rituelle. À l'instar des peintures corporelles, il s'agit d'une forme d'expression artistique éphémère. Alors que ces peintures sur le sol ne sont plus effectuées aujourd'hui, les peintures corporelles gardent quant à elles toute leur importance dans les performances rituelles ${ }^{9}$.

En plus des motifs et dessins rituels kuruwarri, il existe des formes et des pratiques graphiques à caractère public et quotidien, les dessins sur le sable (walkala, « sand drawings »). Cette forme d'art narratif ${ }^{10}$ est essentiellement éphémère en ce que le dessin ne dure que le temps de la narration du récit. On retrouve dans cette pratique cette étroite relation et complicité entre récits et dessins, comme si ce qui est raconté devait en quelque sorte être prolongé et inscrit sur le sol. Les dessins sur le sable sont une pratique très répandue et valorisée dans les régions désertiques, effectués par tout un chacun, sans distinction d'âge ou de genre. Tout est prétexte à raconter une histoire laquelle sera accompagnée d'un dessin sur le sable : une journée en brousse pour les activités de chasse et de cueillette ; un séjour en ville ; un événement récent ou passé ; 
un arrangement rituel ; un rêve ; la disposition d'un campement ; un épisode Tjukurrpa dans sa forme publique, etc.

Entre eux ou lorsqu'ils s'adressent aux plus jeunes et aux enfants, les adultes et les aînés vont régulièrement accompagner leurs récits d'un dessin sur le sable, et ceci peu importe le lieu où ils se trouvent : dans la communauté, dans un campement ou en brousse. Comme l'art de creuser, les dessins sur le sable exigent une posture corporelle et des mouvements particuliers. L'orateur est assis sur le sol; dans un premier temps, il prend soin de nettoyer et d'aplanir une surface avec le plat de la main sur le sol sablonneux devant lui. Le sable n'est-il pas, comme le fait remarquer Watson, une toile à jamais disponible qui peut être utilisée et effacée à volonté, de jour comme de nuit (2003 : 74). Avec sa main (la main droite généralement) et ses différentes parties, ses doigts, ou bien à l'aide d'un mince bâton légèrement recourbé (milpa), au fur et à mesure que se déroule le récit, le narrateur trace sur le sable les différents motifs indiquant les principaux éléments qui composent le récit.

Parmi les principaux motifs notons : les cercles, demi-cercles et cercles concentriques, les lignes droites, courbes ou serpentées, les points ou encore les empreintes des espèces animales. Chaque motif est nécessairement plurivoque et ne prend sa signification contextuelle qu'au sein de l'agencement global et dans sa relation avec le récit. Un cercle, par exemple, peut signifier un campement, un point d'eau, un arbre ou une colline ; un demi-cercle indique souvent une personne assise ; les lignes, droites ou serpentées, indiquent généralement des chemins et un déplacement d'un lieu à un autre ; un simple trait signifie un objet tel un bâton à fouir ou un javelot (Voir aussi Munn 1973). En s'inspirant de l'approche sémiotique de C. S. Peirce, Watson (2003) a clairement souligné la dimension et la valeur iconiques, symboliques et indicielles de ces expressions graphiques. Dès que le récit est terminé, le narrateur prend soin d'effacer le dessin, avec un geste délicat de la main. Ceci afin d'éviter que quiconque, absent lors de la narration et de l'exécution du dessin, ne soit tenté, a posteriori, d'interpréter le dessin.

Les dessins éphémères sur le sable sont une partie intégrante de l'événement narratif et un médium très valorisé afin de communiquer une expérience, d'instruire les enfants et de transmettre tout un ensemble d'informations : cartes et lieux sur le territoire ; relations de parenté ; segments mythiques ; empreintes animales ; techniques de chasse et de cueillette; préparation des produits de la chasse et de la cueillette, etc. Le récit, l'événement narré ou la transmission d'une connaissance doit en quelque sorte être inscrit sur le sol sablonneux, de manière éphémère, de la même façon que les êtres Tjukurrpa ont inscrit le sol et le paysage, mais de manière plus permanente.

\section{Exprimer le désert en acrylique}

L'art de raconter et les différentes formes traditionnelles d'expression graphique (motifs kuruwarri, peintures corporelles, dessins sur le sable) ont trouvé leur expression contemporaine dans les peintures acryliques. Dès la fin des années 1970, la majorité des communautés aborigènes du Désert occidental a été traversée par ce qu'il est maintenant convenu d'appeler le mouvement acrylique. Les Aborigènes se sont approprié des matériaux 


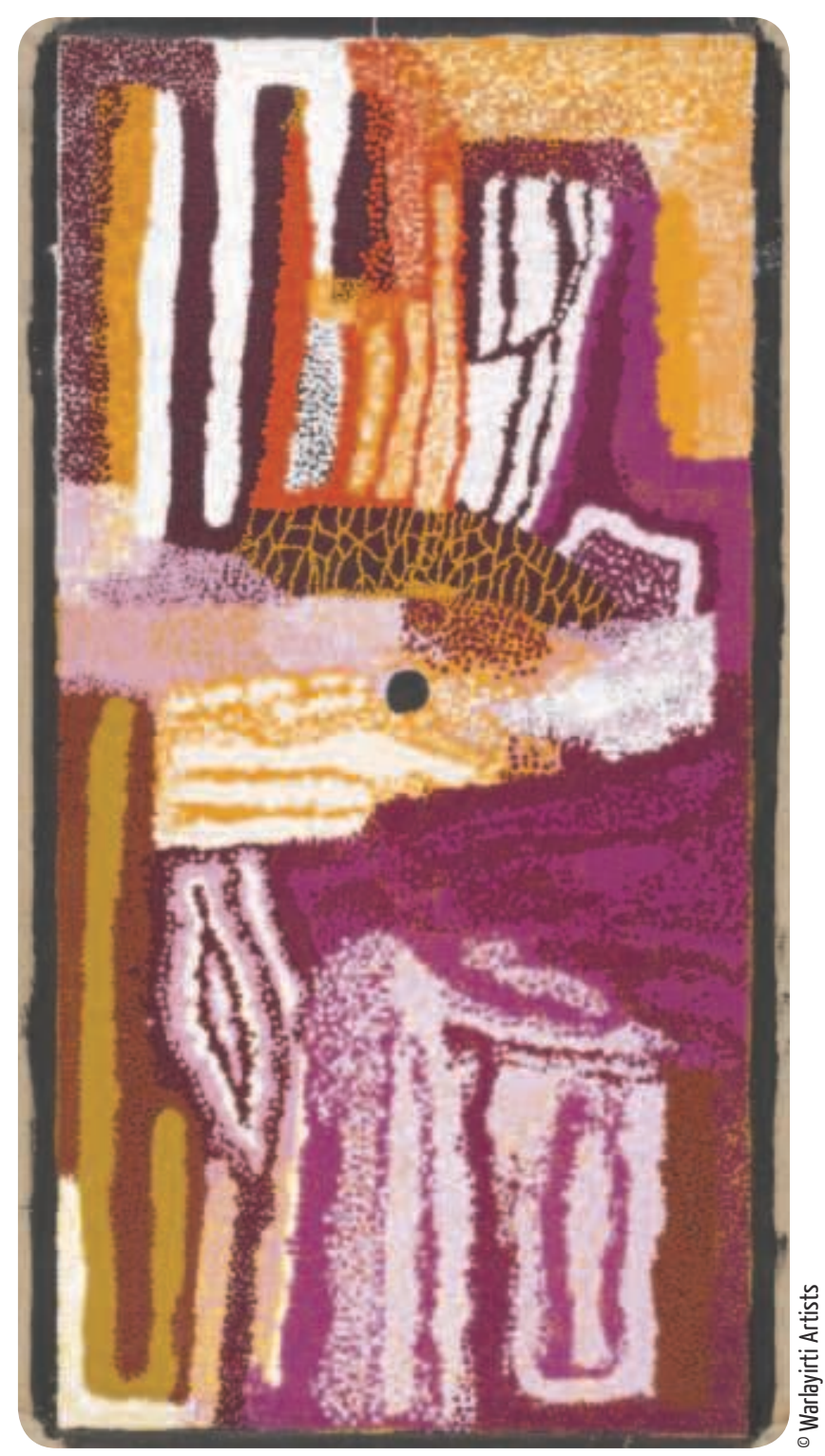

Ci-dessus, Winpurpula par Christine Yukenbarri

L'artiste a peint le « pays » de sa mère situé au sud de Balgo. Le cercle au centre représente un point d'eau permanent ; les lignes foncées évoquent les dunes de sable, très présentes dans la région; des produits de la cueillette, tels les graines lukararra et les fruits sauvages kumpupatja, y sont aussi représentés. Acrylique sur toile, $150 \times 74,5 \mathrm{~cm}, 2007$. Reproduit avec la permission de Warlayirti Artists. modernes, l'acrylique et la toile rectiligne, afin de continuer à raconter leurs récits mais aussi à exprimer autrement le désert, et à partager ceux-ci avec les non-Aborigènes. En effet, cette nouvelle forme d'expression artistique a connu un essor considérable sur le marché artistique international (Myers 2002). Dès le départ, les Aborigènes ont réalisé que l'acrylique - par son caractère permanent mais aussi avec toute sa palette de couleurs - leur offrait de nouvelles possibilités et ils ont ainsi décidé d'en explorer toutes les potentialités : esthétiques, politiques, dialogiques et cosmologiques.

Dans le contexte des communautés sédentaires où les gens n'occupent plus les territoires de manière permanente, les peintures acryliques représentent très certainement une façon de confirmer et d'entretenir le lien aux territoires et de maintenir les relations avec les êtres Tjukurrpa qui habitent les lieux. Le geste de peindre - au même titre que les danses et les chants rituels - contribue aussi à la reproduction des ressources animales, végétales et ancestrales du territoire. Alors que les jeunes d'aujourd'hui n'ont pas toujours la possibilité de visiter les territoires ancestraux, les peintures acryliques sont un moyen de transmission des savoirs mythiques, géographiques, écologiques et familiaux. La production des peintures se fait majoritairement dans les campements ; ceux qui peignent, hommes et femmes, sont toujours très généreux dans les histoires qu'ils transmettent, et celles-ci débordent très largement ce qui est représenté sur la toile. En outre, la vente des peintures génère, pour plusieurs familles de la communauté, des revenus d'appoint nonnégligeables. Notons aussi qu'à l'instar des dessins sur le sable, les peintures acryliques sont effectuées alors que les artistes sont assis à même le sol, valorisant dès lors un plan horizontal plutôt que vertical ${ }^{11}$

Chaque peinture est nécessairement identifiée à un «pays » (ngurra) auquel l'artiste est affilié, évoquant ainsi les épopées des êtres Tjukurrpa, des lieux le long des tracés de géographie mythique et des réseaux sociaux. Une personne n'est autorisée à peindre que les « pays », soit les lieux auxquels elle est affiliée selon la Loi ancestrale. Les sources d'inspiration sont multiples : les récits Tjukurrpa liés au(x) lieu(x) représenté(s), la configuration du terrain, les ressources animales et végétales, les expériences passées ou récentes du peintre ou des membres de sa famille élargie en ces lieux. Chaque peinture raconte une histoire et peut se « lire » tel un agencement de récits mythiques, d'histoires familiales, d'expériences personnelles et de 
savoirs topographiques, géographiques, hydrologiques et écologiques. Les quatre peintures sélectionnées ont été produites par des femmes. Chaque artiste y a peint un « pays » (ngurra) auquel elle est affiliée et avec lequel elle entretient une relation intime ; un pays, dominé par les plaines et les dunes de sable et de spinifex. Ces toiles évoquent admirablement la connaissance topographique et écologique du désert, les ressources végétales disponibles ainsi que les liens avec des êtres et des récits Tjukurrpa.

Une autre forme d'expression artistique a vu le jour à Balgo au début des années 1980, faite de sculptures de grès (« sandstone »). Au contraire de la peinture acrylique, cette forme fut sans lendemain. De 1980 à 1982, des hommes et des femmes se sont investis dans la production de ces sculptures, représentant surtout des animaux et des récipients familiers aux Aborigènes. Il est probable

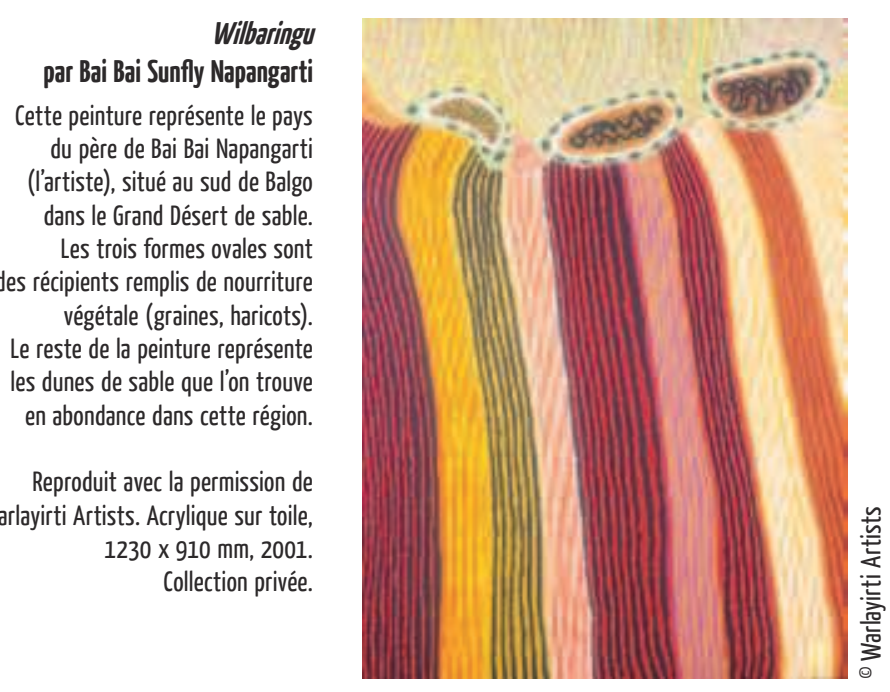

\section{Martumili Ngurra par Kumpaya Girgaba, Jakayu Biljabu, Ngamaru Bidu, Thelma Judson, Nola Taylor et Jane Girgaba}

Cette peinture raconte le Rêve des Sept Sœurs (Minyipuru), au « pays » des Martu, dominé par les plaines et les dunes de sable recouvertes de spinifex. Les gradations de couleur évoquent les cinq étapes de régénérescence de la végétation qui font suite à la pratique de brûlis en mosäquue.

Reproduit avec la permission de Martumili Artists. Acrylique sur toile, 324 x 508 cm., 2009. Collection du National Museum of Australia.

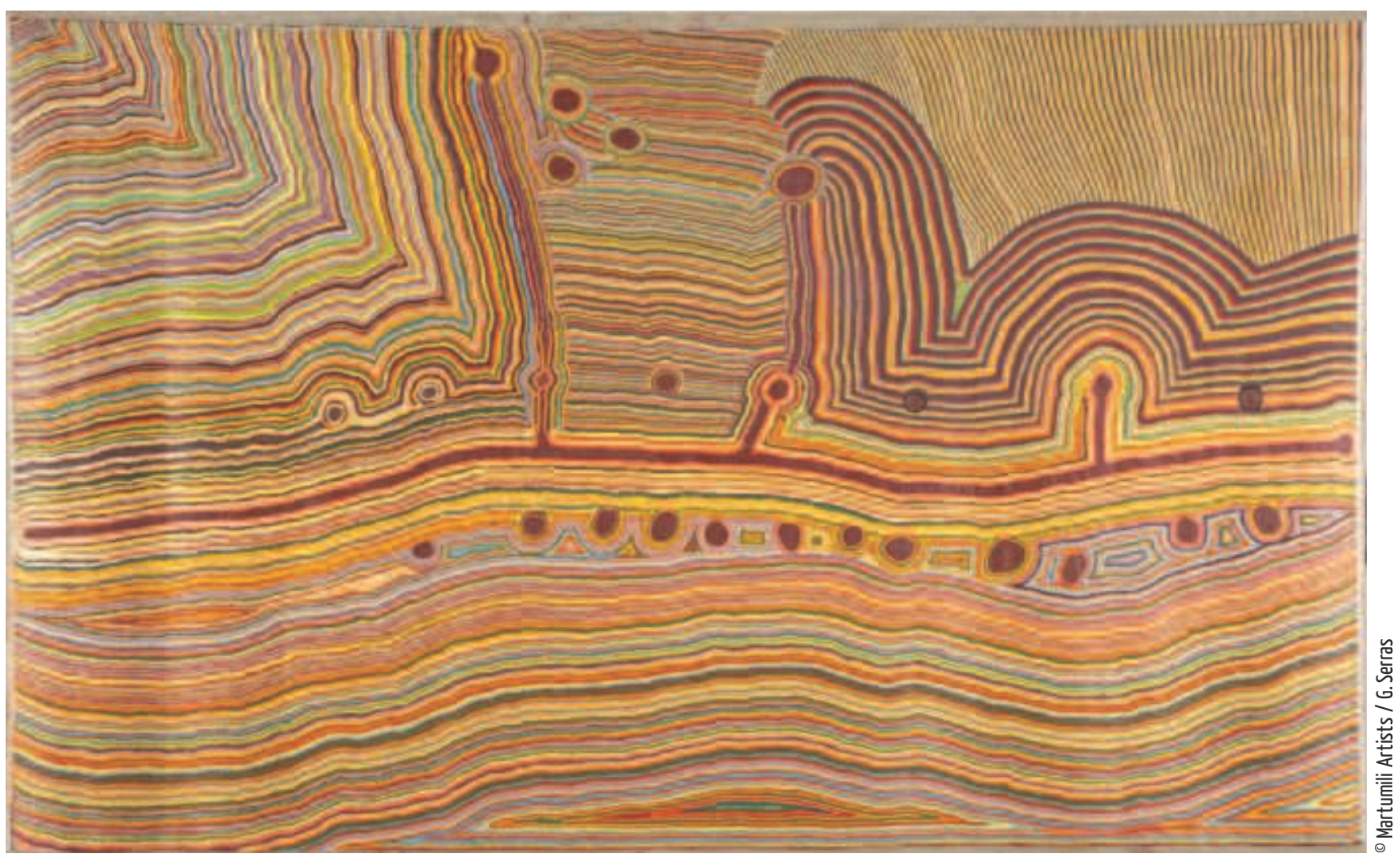


que de telles sculptures aient été produites traditionnellement, mais à petite échelle et à des fins rituelles. Durant ces deux années, la production fut très abondante; nous visitions régulièrement la carrière à une centaine de kilomètres au sud de Balgo afin de renouveler le stock de pierres de grès brutes. Les Aborigènes espéraient les vendre et en tirer quelques profits. Mais l'artiste le plus prolifique, un homme dans la quarantaine, est décédé, en l'espace de quelques mois, des suites d'un cancer. La communauté interpréta alors sa maladie comme un mauvais présage lié à la production des sculptures. D'un commun accord, ils mirent fin à celle-ci. À ce jour, l'interdit a été respecté. Au même moment, la peinture acrylique fit son apparition dans la communauté et n'a cessé dès lors de se développer.

\section{$\&$}

Dans cet environnement où domine le sable, et suivant la perspective et l'expérience des Aborigènes, celui-ci ne saurait être appréhendé sans considérer les autres éléments naturels, et parmi ceux-ci, le vent, l'eau et le feu. Étonnamment, et bien que le sable soit une présence incontournable dans le monde des Kukatja et de leurs voisins, ils ont peu de mots pour le désigner. Le dictionnaire de la langue kukatja (Valiquette 1993) n'en recense que deux, soit parna et walya, deux termes génériques utilisés aussi pour désigner la poussière, le sol ou la terre. Par ailleurs, ces maîtres dans l'art de pister et de repérer les différentes traces animales et végétales sur le sol sablonneux possèdent un vocabulaire très riche pour désigner tous les types d'empreintes; il en va de même du vocabulaire pour identifier les différents types de nuage, de pluie et de phénomènes climatiques.

Dans le désert se côtoient les «formes de la permanence », un terme que Stanner (1979) avait utilisé pour désigner les ressources matérielles, spirituelles et rituelles léguées par les êtres du Rêve, mais aussi, et comme nous l'avons vu, celles de l'impermanence. Les traces et empreintes balayées par le vent, les mini-tornades instantanées, les pluies incertaines et irrégulières, les plaines de spinifex brûlées par le feu en quelques instants, les peintures corporelles et les dessins sur le sable ou encore les campements et les coupe-vents temporaires sont autant de réalités et de pratiques qui évoquent l'éphémère et l'impermanence.

Au fil de leur longue histoire, les Aborigènes ont développé une connaissance intime et approfondie de l'écologie fragile et de la biodiversité de leur environnement désertique. Le découvrir et le parcourir avec eux est toujours une expérience enrichissante, notamment lorsqu'il s'agit des activités de chasse et de cueillette ou des récits évoquant les pérégrinations des êtres du Rêve en tel ou tel lieu. Or, là comme ailleurs, les savoirs traditionnels et leur transmission intergénérationnelle sont sujets à une érosion graduelle. Bien que les jeunes générations continuent de mettre en pratique certains des savoirs de leurs aînés comme le repérage et la lecture des pistes animales ou les dessins sur le sable, force est d'admettre que nombre de savoirs et pratiques sont aujourd'hui tombés en désuétude. 


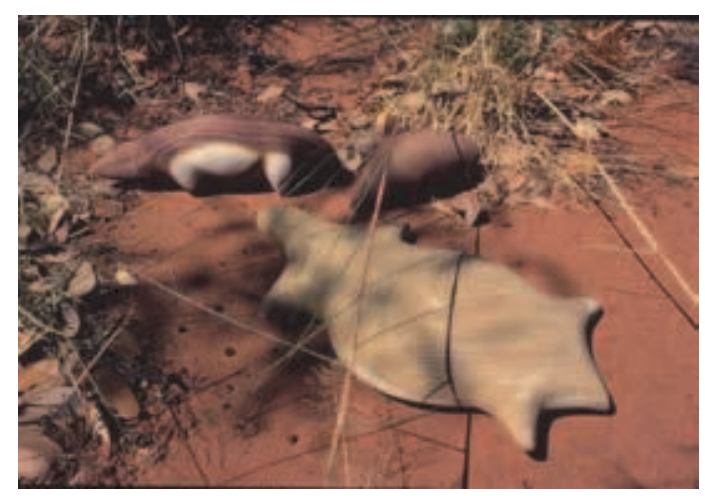

Sculptures de grès, 1980-1981,

Balgo

Artistes inconnus
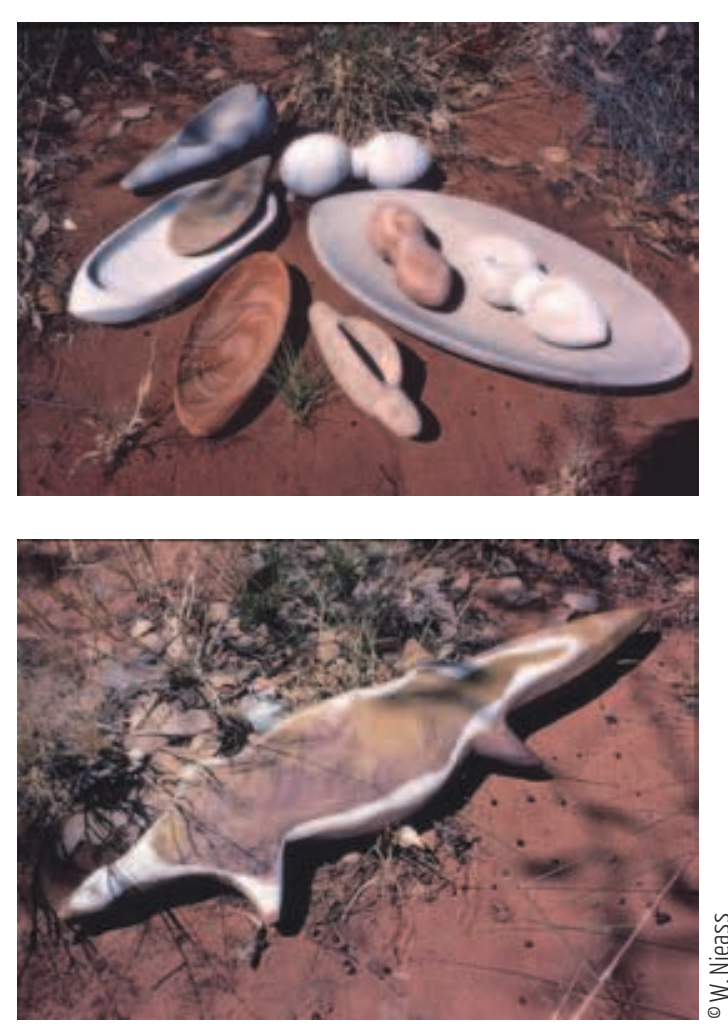

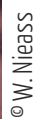




\section{NOTES}

Photo d'ouverture: Nyilla par Eubena Nampitjin. Cette peinture du « pays » de l'artiste, au sud de Balgo, évoque le Rêve des Femmes Bâtons à fouir (Kanaputa) et les savoirs rituels qui leur sont associés, ainsi que les produits et les activités de la cueillette. Acrylique sur toile, 100 x 76 cm, 1989. Collection privée. Reproduit avec la permission de Warlayirti Artists.

Toutes les illustrations sont de l'auteur sauf mention contraire.

1. Cette communauté est relativement isolée. Halls Creek, la ville la plus proche, est située à $240 \mathrm{~km}$ au nord.

2. Ces appellations ne réfèrent pas à des « tribus », mais plutôt à des entités linguistiques, culturelles et territoriales fluides et plus ou moins circonscrites. Il est fréquent d'ailleurs qu'une même personne se réclame de différentes appellations en référence, entre autres, à l'un ou l'autre de ses grands-parents.

3. Parmi ces objets, les boomerangs sont encore fabriqués. Bien qu'ils ne soient plus utilisés pour la chasse, ils servent à battre la mesure lors des chants rituels ou sont utilisés comme objets d'échange.

4. Un espace fraîchement brûlé attirera les outardes australiennes à la recherche d'insectes, alors que les jeunes pousses sont attrayantes pour les kangourous.

5. Le terme mangarri désigne l'ensemble des nourritures végétales: tubercules, graines, fèves, nectars et fruits sauvages de toutes sortes, et inclut aussi les larves et insectes consommés.

6. C'est à ma demande que des femmes ont accepté, à quelques occasions, de reproduire ce processus de collecte et de préparation.

7. L'accessibilité, la divulgation et la transmission des savoirs mythiques et rituels relèvent de règles à la fois strictes et complexes. Voir, parmi d'autres, Poirier (2001), Dussart (2000) pour les Warlpiri du désert central, Myers (1986) pour les Pintupi, Tonkinson (1991) pour les Martu.
8. L'ocre rouge est particulièrement prisé, directement associé au pouvoir des êtres Tjukurrpa ; plus sa brillance est prononcée, plus grand est son pouvoir. L'ocre rouge est aussi utilisé à des fins médicinales : broyé et enduit sur le corps, durant la saison chaude, il atténue la chaleur ; broyé et mélangé avec de la salive, il est appliqué sur des plaies ; broyé et mélangé à du gras animal, il est utilisé pour masser les parties du corps affectées par une maladie (Peile 1997 : 229-231).

9. Les principaux rituels encore pratiqués à Balgo et dans la région sont les rites funéraires et initiatiques.

10. L'ouvrage classique de Munn (1973) nous avait initiés aux dessins sur le sable et à l'iconographie des Warlpiri (Désert central). Pour Balgo, cette pratique a été documentée par Watson dans Piercing the Ground (2003). Eickelkamp, qui a conduit des recherches à Ernabella, une communauté aborigène du Désert central, a noté que cette pratique est aussi particulièrement répandue et valorisée chez les jeunes filles (2011).

11. Les Aborigènes s'amusent d'ailleurs de ce que les acheteurs de leurs œuvres suspendent celles-ci sur les murs des musées et de leurs maisons. 


\section{REMERCIEMENTS}

Je remercie Sébastien Boulay et Marie-Luce Gélard de m’avoir invitée à participer à ce numéro de la revue Techniques \& Culture. Depuis les années 1980, mes recherches et mes séjours dans le Désert occidental australien ont été rendus possibles grâce au support financier du Conseil de recherches en sciences humaines du Canada. Je le remercie de son soutien et de la confiance qu'il m'a toujours témoignée. J'adresse enfin mes plus sincères remerciements aux familles de Balgo, pour leur hospitalité et leur générosité au fil des décennies, et envers lesquelles je suis à jamais redevable.

\section{POUR CITER CET ARTICLE}

Poirier, S. 2014 Traces, bâtons à fouir et dessins sur le sable chez les Aborigènes du Désert occidental australien, in S. Boulay \& M.-L. Gélard, Vivre le sable! Corps, matière et sociétés, Techniques \& Culture 61 : 144-165.

\section{RÉFÉRENCES}

Bauman, R. 1986 Story, Performance and Event. Cambridge : Cambridge University Press.

Bird, D., Bird., R. \& Parker, C. 2005 Aboriginal Burning Regimes and Hunting Strategies in Australia's Western Desert. Human Ecology, 33 (4) : 443-464.

Cane, S. 1984 Desert Camps : A case Study of Stone Artifacts and Aboriginal Behavior in the Western Desert. Canberra : Australian National University (Thèse de doctorat).

Cane, S., Stanley, O. 1985 Land use and resources in desert homelands. Darwin : Australian National University, North Australian Research Unit.

Dussart, F. 2000 The Politics of Ritual in an Aboriginal Settlement. Kinship, Gender, and the Currency of Knowledge. Washington: Smithsonian Institution Press.

Eickelkamp, U. 2011 Sand Storytelling : Its Social Meaning in Anangu Children's Lives. In U. Eickelkamp (dir.), Growing up in Central Australia. New Anthropological Studies of Aboriginal Childhood and Adolescence. New York : Berghahn : 103-130.

Munn, N. 1986 [1973] Walbiri Iconography. Chicago : Chicago University Press.

Myers, F. 2002 Painting Culture. The Making of an Aboriginal High Art. Durham : Duke University Press.

- 1986 Pintupi Country, Pintupi Self : Sentiment, Place and Politics among Western Desert Aborigines. Washington, DC : Smithsonian Institution Press.

Peile, A. 1997 Body and Soul. An Aboriginal View. Carlisle (Western Australia) : Hesperian Press. 
Poirier, S. 2004 Ontology, Ancestral Order, and Agencies among the Kukatja (Australian Western Desert). In J. Clammer, S. Poirier and E. Schwimmer (dir.) Figured Worlds. Ontological Obstacles in Intercultural Relations. Toronto : University of Toronto Press.

- 2001 Les Politiques du savoir rituel. Réflexions sur les relations de genre chez les Kukatja (Désert occidental australien). In Sexe relatif ou sexe absolu? De la distinction de sexe dans les sociétés. Eds. Catherine Alès and Cécile Barraud, 111-134. Paris : Éditions de la Maison des sciences de l'homme.

- 1996 Les Jardins du nomade. Cosmologie, territoire et personne dans le désert occidental australien. Münster : Lit Verlag.

Stanner, W. E. H. 1979 White Man Got no Dreaming. Essays 1938-1973. Canberra : Australian National University Press.

Tonkinson, R. 1991[1978] The Mardu Aborigines. Living the Dream in Australia's Desert. New York : Holt, Rinehart \& Winston.

Vaarzon-Morel, P., Gabrys, K. 2009 Fire on the horizon : contemporary Aboriginal burning issues in the Tanami Desert, Central Australia. Geojournal 74 : 465-476.

Valiquette, H. (dir.) 1993 A Basic Kukatja to English Dictionary. Wirrimanu : Luurnpa Catholic School.

Walsh, F. J. 1990 An Ecological Study of Traditional Aboriginal Use of Country: Martu in the Great and Little Sandy Deserts, Western Australia. Proceedings of the ecological society of Australia 16:23-37.

Watson, C. 2003 Piercing the Ground. Balgo women's image making and relationship to country. Perth : Fremantle Arts Centre Press. 


\section{RÉSUMÉ}

Traces, bâtons à fouir et dessins sur le sable chez les Aborigènes du Désert occidental australien. Cet article dresse un portrait d'ensemble de la relation intime que les Kukatja, un groupe aborigène du désert occidental australien, entretiennent avec leur environnement où dominent les plaines et les dunes de sable, et considéré parmi les plus inhospitaliers de la planète. Traditionnellement chasseurs-cueilleurs nomades et sédentarisés à partir des années 1950, les Kukatja habitent aujourd'hui la petite communauté de Balgo, sise aux confins nord du désert de Gibson. Dans le contexte actuel et lorsqu'ils en ont la possibilité, ils visitent leurs territoires ancestraux afin, entre autres, d'y pratiquer leurs activités de chasse et de cueillette. Dans le monde des Aborigènes, les ressources matérielles et spirituelles, les savoirs écologiques et mythiques sont étroitement interreliés, tous émanent du Tjukurrpa, le Rêve. L'article souligne à la fois la diversité et la rareté des ressources animales et végétales et présente, suivant le cycle saisonnier, différentes pratiques de collecte et de préparation. La question des traces est aussi abordée : les traces permanentes laissées par les êtres du Rêve et les traces évanescentes qui marquent l'étendue du sol désertique. Les Aborigènes sont passés maîtres dans l'art de lire tous les types d'empreintes. À l'art de pister s'ajoute l'art de creuser le sol sablonneux, à la recherche d'eau, mais aussi de reptiles et de tubercules. De bois ou de métal, le bâton à fouir (wana) est l'outil privilégié et indispensable des femmes. Les traces, ce sont aussi les dessins éphémères sur le sable, exécutés lors de la narration de divers types de récits, ou encore les peintures à l'acrylique dont le commerce est aujourd'hui florissant.

\section{ABSTRACT}

Traces, digging sticks and sand drawings among Aboriginal people of the Australian Western Desert. This article presents an overall picture of the intimate relationship that the Kukatja, an Aboriginal group of the Australian Western Desert, maintain with their environment, dominated by sand plains and dunes, and considered to be one of the most inhospitable on the planet. Former nomadic hunters and gatherers and sedentarized from the 1950's onwards, the Kukatja live today in Balgo, a small community at the northern fringe of the Gibson Desert. In today's context and whenever they have the possibility, they visit their ancestral territories in order, among others, to practice their hunting and gathering activities. In the world of the Aborigines, material and spiritual resources, ecological and mythical knowledge are closely intertwined, all come from the Tjukurrpa, the Dreaming. The article underlines both the diversity and scarcity of animal and plant resources and presents, according to the seasonal cycle, various practices of gathering and preparation. The question of traces is also discussed: the permanent traces left by the Dreaming Beings and the evanescent traces found all over the desert soil. The Aborigines have mastered the art of reading all types of tracks. To the art of tracking we must add the art of digging into sandy soil, in search of water, but also of reptiles and tubers. The digging stick (wana), made of wood or metal, is the women's privileged and indispensable tool. By traces, we also mean the impermanent sand drawings performed during the narration of various types of stories, as well as the acrylic paintings for which there are now a flourishing commerce.

\section{MOTS CLÉS}

Désert australien, Aborigènes, chasseurs-cueilleurs, pistes, bâton à fouir, dessins sur le sable

\section{KEYWORDS}

Australian desert, Aborigines, hunter-gatherers, tracks, digging stick, sand drawings 